\title{
ON THE SOLVABILITY OF NONLINEAR DIFFERENTIAL EQUATIONS SUBJECT TO GENERALIZED BOUNDARY CONDITIONS
}

\author{
Benjamin FreEdman And Jesús RodrígueZ
}

Abstract. In this paper, we analyze nonlinear differential equations subject to generalized boundary conditions. More specifically, we provide a framework from which we can provide conditions, which are straightforward to check, for the solvability of a large number of nonlinear scalar boundary value problems. We begin by giving our general strategy which involves the reformulation of our boundary value problem as an operator equation. We then proceed to establish our results and compare them to closely related previous work.

Mathematics subject classification (2010): 34A34, 34B15, 47H09, 47H10, $47 \mathrm{~J} 07$.

Keywords and phrases: Boundary value problems, ordinary differential equation, nonlinear equations, fixed-point theorems.

\section{REFERENCES}

[1] B. AhmAD, J. J. NiETo, Existence of solutions for nonlocal boundary value problems of higher-order nonlinear fractional differential equations, Abstract and Applied Analysis 2009, (2009).

[2] A. Boucherif, Nonlinear three-point boundary value problems, J. Math Anal. Appl. 77, 2 (1980), $577-600$.

[3] K. BRown, Nonlinear boundary value problems and a global inverse function theorem, Annali di Matematica Pura ed Applicata 106, 1 (1975), 205-217.

[4] K. BRown, S. Lin, Periodically perturbed conservative systems and a global inverse function theorem, Nonlinear Anal.: Theory, Methods \& Applications 4, 1 (1980), 193-201.

[5] P. Drábek, J. Milota, Methods of Nonlinear Analysis: Applications to Differential Equations, Birkhäuser Verlag AG, Basel, 2007.

[6] R. MA, Nonlinear discrete Sturm-Liouville problems at resonance, Nonlinear Anal.: Theory, Methods \& Applications 67, 11 (2007), 3050-3057.

[7] D. Maroncelli, Scalar multi-point boundary value problems at resonance, Differential Equations and Applications 7, 4 (2015), 449-468.

[8] J. RODRÍGUEZ, Galerkin's method for ordinary differential equations subject to generalized nonlinear boundary conditions, J. Differential Equations 97, 1 (1992), 112-126.

[9] J. RodRÍGUEZ, Nonlinear differential equations under Stieltjes boundary conditions, Nonlinear Anal.: Theory, Methods \& Applications 7, 1 (1983), 107-116.

[10] J. RodrígueZ, Nonlinear discrete Sturm-Liouville problems, J. Math Anal. Appl. 308, 1 (2005), 380-391.

[11] J. Rodríguez, Z. Abernathy, Nonlinear discrete Sturm-Liouville problems with global boundary conditions, Journal of Difference Equations and Applications 18, 3 (2012), 431-445.

[12] J. RodrígueZ, Z, ABERnAthy, On the solvability of nonlinear Sturm-Liouville problems, J. Math Anal. Appl. 387, 1 (2012), 310-319.

[13] J. RodrígueZ, A. J. SuAREZ, On nonlinear perturbations of Sturm-Liouville problems in discrete and continuous settings, Differential Equations and Applications 8, 3 (2016), 319-334.

[14] J. RODRÍGUEZ, P. TAYLOR, Multipoint boundary value problems for nonlinear ordinary differential equations, Nonlinear Analysis: Theory, Methods \& Applications 68, 11 (2008), 3465-3474.

[15] M. URABE, Galerkin's prodcedure for nonlinear periodic systems, Archive for Rational Mechanics and Analysis 20, (1965), 120-152. 